\title{
Estudio comparativo para propagación vegetativa de Chondracanthus chamissoi "Yuyo" sobre tres tipos de sustrato en ambiente controlado y su viabilidad en la región Moquegua
}

\section{(Comparative study for vegetative propagation of Chondracanthus chamissoi "Yuyo" on three types of substrate in a controlled environment and its viability in the Moquegua region)}

Jose Carlos Zapata-Rojas', Alejandro Marcelo Gonzales-Vargas², Sheyla Amanda Zevallos-Feria

\section{Resumen}

La investigación compara la propagación vegetativa de Chondracanthus chamissoi, conocida también como "Yuyo" en Perú, en tres tipos diferentes de sustrato, bajo condiciones controladas en laboratorio. Los sustratos usados para la comparación fueron; TA; valva de almeja, TB; Malla de fruta, TC; cabo de propileno y TP; concha de Concholepas concholepas. Según el estudio, existió mejor supervivencia en TB, determinando la formación de (DFS) a partir de 20 a 23 días. Según los resultados, la estrategia de propagación vegetativa es viable para el escalamiento comercial en el litoral de Moquegua, Perú.

\section{Palabras Clave}

Macroalga; C. chamissoi; crecimiento vegetativo; supervivencia; DFS (Discos de fijación secundaria).

\begin{abstract}
The research compares the vegetative propagation of Chondracanthus chamissoi, commonly called "Yuyo" in Peru on three different types of substrate, under laboratory controlled conditions. The substrates used for comparison were; TA; clam leaflet, TB; Fruit mesh, CT; cape of propylene and TP; Concholepas concholepas shell. According to the study, there was better survival in TB; determining the formation of (DFS) from 20 to 23 days, according to the results, the vegetative propagation strategy is viable for commercial escalation in the coast of Moquegua, Peru.
\end{abstract}

Keywords

Macroalgae; C. chamissoi; vegetative growth; survival; DFS (Secondary Fixing Disks).

\section{Introducción}

Chondracanthus chamissoi es una especie autóctona y propia de la costa templada del Pacífico sur, que se dispone desde Paita-Perú hasta Chiloé-Chile y reside en las áreas rocosas del intermareal y submareal. En la actualidad, las algas están consideradas dentro del grupo de súper alimentos, debido a sus elevadas propiedades nutritivas.

Según información de la FAO (2018b), en el año 2016 la producción mundial de algas marinas llegó a alrededor de 30.8 millones de toneladas, de las cuales el 98 \% de la producción provenía de la acuicultura y el $2 \%$ restante provenía de la cosecha de camas naturales. La contribución de América Latina solamente llegó al 1.2 \% de esta producción (373 mil toneladas), donde

$1 \quad$ Universidad Nacional de Moquegua, Perú. [zapatarojasjosecarlos@gmail.com, http://orcid.org/0000-0001-5050-4512]

2 Universidad Nacional de Moquegua, Perú. [alejandromgonzales@outlook.com, https://orcid.org/0000-0002-3256-907X]

3 Instituto del Mar del Perú, Perú. [amandazevallosferia@gmail.com, https://orcid.org/0000-0001-8964-5240] 
la aportación del país chileno representa el $88 \%$. Mientras que la producción restante se divide entre Perú con 15293 toneladas (4.1\%) y México con 13801 toneladas (3.7 \%). Con respecto al cultivo de algas, el principal productor para Latinoamérica es Chile, siendo abastecedor del $95 \%$ de la producción (14 846 toneladas). Le siguen Brasil (4.68 \%), México (1.15\%), Ecuador (0.03\%) y Perú $(0.01 \%)$. Actualmente la demanda de $C$. chamissoi es alta y sigue en crecimiento.

La actividad extractiva y recolección del recurso hidrobiológico en los últimos años se ha incrementado rápidamente en el litoral sur del Perú (Delgado, E. y Chang, F., 2015; Imarpe, 2010), evidenciándose una creciente demanda en el mercado nacional e internacional. El principal destino para la producción de algas peruanas es el mercado asiático, con precios de US\$ 11 FOB por kilo, figurando entre las más cotizadas luego de las algas pardas al "yuyo" C. chamissoi. Por lo tanto, en Perú y en otros países abastecedores del producto, existe un estricto estándar de calidad que engloba cualidades como: limpieza, libre de epífitas e impurezas, carencia de estructuras reproductivas, color y textura bien definidos.

Si el total de la producción se abasteciera solo de praderas naturales, sería difícil entregar cantidades suficientes de productos de calidad en un programa de producción confiable. Por este motivo, la acuicultura se visualiza como una de las alternativas más eficientes para la solución de este problema.

Bajo ese contexto, varios expertos realizaron investigaciones relacionadas a la propagación vegetativa de $C$. chamissoi, principalmente en Chile, obteniendo resultados atractivos para cultivar.

La implementación de la tecnología de cultivo de C. chamissoi en otras latitudes como en Chile es factible (Colque Arce, L. M., 2017), constituyendo una alternativa en la recuperación de praderas naturales de macroalgas y promoviendo la diversificación de la actividad acuícola para realizar un manejo adecuado de su pesquería; tecnología que incluye cultivos de propagación por esporulación y vía vegetativa. Se destacan experiencias con mejores resultados mediante propagación vegetativa por discos de fijación secundaria en sustrato natural (valvas de moluscos lamelibranquios) (Sáez y Macchiavello,2018), método replicado en este estudio. Se ha observado una notable disminución en las algas cosechadas en Chile, pasando de 25000 t en el año 2000 a solo 977 t en el año 2010 (Sernapesca, 2011).

Esto ha incrementado la presión extractiva de este preciado recurso hidrobiológico marino; en consecuencia, se busca alternativas para solucionar este problema, a través de la adaptación de volúmenes tecnológicos para desarrollar el cultivo de macroalgas; por lo que se deben efectuar estudios relacionados con la tecnología de propagación de "yuyo" C. chamissoi y validarlo en la región de Moquegua, con la finalidad de no depredar esta especie en beneficio del ecosistema marino y de las comunidades pesqueras asentadas en el litoral.

Por este motivo, la metodología de propagación vegetativa avizora una tendencia prometedora para el desarrollo de cultivo de C. chamissoi en el litoral marino peruano.

La investigación tiene como objeto primordial realizar un análisis de comparación de propagación vegetativa en tres tipos diversos de sustrato en ambiente controlado para la especie C. chamissoi, en el litoral de la región Moquegua, con la implementación del cultivo en laboratorio, la evaluación del periodo de formación de DFS y variables de crecimiento en longitud.

\section{Metodología}

El estudio se llevó a cabo en un ambiente controlado de las instalaciones del laboratorio de investigación acuícola del Instituto del Mar de Perú, sede llo. 
El modelo de análisis se basó en un diseño experimental totalmente al azar, con tres repeticiones de tres diferentes tipos de tratamientos, resultando un total de nueve unidades experimentales. Los sustratos utilizados fueron las Valvas de Almeja, Mallas de fruta, cabo de polipropileno y la concha de chanque.

Los tratamientos empleados fueron: Tratamiento A: constituido por el cultivo de C. chamissoi mediante propagación vegetativa a partir de discos secundarios en Valvas almeja. Tratamiento B: constituido por el cultivo de $C$. chamissoi mediante propagación vegetativa a partir de discos secundarios en Malla de fruta. Tratamiento C: constituido por el cultivo de C. chamissoi mediante propagación vegetativa a partir de discos secundarios en cabo polipropileno. Tratamiento P: grupo control de C. chamissoi en concha de chanque. Posteriormente se usó el Método Gulland y el Modelo Ricker, sobre la base de las valoraciones del análisis comparativo experimental para obtener la evaluación de los factores de propagación y supervivencia de C. chamissoi.

En lo concerniente a las etapas de la experimentación se inició con la recolección de $C$. chamissoi (10 kg.) en los márgenes de la región de Moquegua, su medio natural de producción. Una vez colectadas, las frondas de láminas vegetativas juveniles fueron trasladadas en una caja isotérmica al laboratorio de Investigación Acuícola del Imarpe llo.

En el laboratorio, al material biológico (C. chamissoi) se le realizó un tratamiento con agua potable y agua de mar micro filtrada, hasta $1 \mu \mathrm{m}$, y se lo acondicionó en un tanque de fibra de vidrio contentivo de 200 litros de agua de mar estéril (micro filtrada hasta $1 \mu \mathrm{m}$ e irradiada con luz ultravioleta), provisto con una piedra difusora para el suministro de aire moderado. Las condiciones del ambiente se mantuvieron a $18{ }^{\circ} \mathrm{C}$ y luz indirecta tenue. Asimismo, el tratamiento del sustrato se llevó a cabo con un lavado natural a las valvas de moluscos, con abundante agua; se desinfectó, utilizando hipoclorito de sodio al $5 \%$, se hizo un enjuague y posteriormente se procedió al secado. Para el caso de sustrato artificial, el tratamiento fue similar. Para el sistema de cultivo se implementó una sala climatizada con estantería distribuida en tres niveles, provista de luz artificial, mediante fluorescentes de 50 watts, conectados a un timer, que controla el encendido y apagado de la luz, según el fotoperiodo previsto y aire suministrado por un regenerador (Blower 2.5 HP).

En cuanto al desarrollo del cultivo, se utilizaron botellas plásticas con capacidad para 7 litros, que se rotularon sobre cada tratamiento. Cada receptáculo incluía 5 litros de agua de mar estéril, que fueron fertilizados con $3 \mathrm{ml}$ del nutriente Provasoli, dos veces por semana (cada 3 días). Fueron colocados $100 \mathrm{~g}$ de "yuyo" por cada envase: (a) para el caso de los sustratos valvas de almeja, las frondas de morfología isomórfica fueron sujetadas con ligas y así se logaron fijar en el sustrato; (b) para la red de fruta, se introdujeron dentro los pedazos fragmentados de algas, con la ayuda de un tubo de policloruro de vinilo, a una pulgada de diámetro; (c) en el sustrato de polipropileno se sujetaron con rafia las frondas; y (d) para el grupo patrón las algas se aseguraron con ligas en valva de chanque. La limpieza del lugar de experimentación se efectuó con base en la técnica del sifoneo y cada 3 días se realizó la sanitización de materiales.

Durante el experimento, una vez acondicionado el material biológico, se evaluó la fijación de DFS y se expresó como porcentaje de los fragmentos adjuntos en relación al número total de fragmentos contenidos en cada botellón, para cada tratamiento. Una vez que se formaron los DFS, las frondas restantes fueron aisladas, evitando ser separadas del sustrato. En cuanto las algas fueron fijadas, se procedió a retirar las frondas que no formaron discos para su posterior monitoreo y cada 10 días se anotó el crecimiento de longitud en las piezas experimentales por cada tratamiento en un ambiente controlado. Y se procedió a medir el crecimiento y supervivencia. El crecimiento se analizó con base en el Método Gulland: 


$$
\mathrm{TC}=(\mathrm{Lti}+1-\mathrm{Lti}) /(\mathrm{ti}+1-\mathrm{ti})
$$

donde:

$\mathrm{TC}=$ Tasa de Crecimiento $(\mathrm{mm}) \mathrm{o}(\mathrm{g})$

$\mathrm{ti}=$ Tiempo (días) de muestreo cero

$\mathrm{ti}+1=$ Tiempo de muestreo subsecuente al ti

Lti = Longitud correspondiente al tiempo ti

Lti+1 = Longitud correspondiente al tiempo ti+1

Y la supervivencia se determinó por el Modelo Ricker:

$\% \mathrm{~S}=\mathrm{Nti} / \mathrm{Nto}$ * 100

donde:

$\% \mathrm{~S}=$ Porcentaje de supervivencia.

$\mathrm{Nti}=$ Número de individuos sobrevivientes al tiempo " $\mathrm{t}$ ".

Nto $=$ Número total de individuos.

En el estudio comparativo se obtuvieron cantidades de cada unidad experimental, que se registraron en hojas de cálculo Excel, para su posterior análisis en el Software estadístico SPSS, versión 21, para determinar la normalidad de las cifras y la homocedasticidad en sus valores de varianzas, aplicando un Anova de una vía $(P=0,05)$. Con ello se aplicó el test de Tukey, comparando medias y contrastando las diferencias en la propagación con respecto a los tratamientos.

\section{Resultados y discusión}

\subsection{Formación de discos de fijación secundarios:}

El tratamiento (A), valvas de almeja sujetado con ligas, se conservó en un periodo de 23 días, con una alternancia de agua cada 7 días. Se adicionó nutriente al medio de cultivo (Provasoli $1 \mathrm{ml}$ / Lt. de agua de mar). La formación de DFS se examinó al día 20 y hacia el día 23 se procedió a retirar el tejido adulto, obteniendo ejemplares jóvenes, que fueron posteriormente registrados.

Para el caso del Grupo control (P), la formación de discos de fijación secundaria posee un tiempo similar al tratamiento (A), asimismo la manipulación durante los días de cultivo que duró el estudio.

Con respecto al Tratamiento (C), durante la primera etapa de estudio, se utilizó cabo de polipropileno como sustrato base para C. chamissoi. Pese a tener el mismo periodo de cultivo que los otros tratamientos y habiendo controlado las condiciones ambientales propias del cultivo, la formación de DFS no se dio. Sin este punto clave, no es posible pasar a la siguiente etapa del cultivo, por lo que a este tratamiento se le consideró no factible para la especie y por lo tanto se dio por finalizado. El uso de cabo de polipropileno fue utilizado por Arbaiza et al. (2019) y lograron la fijación de C. chamissoi, sin embargo para ello tuvieron que tratar el mismo con bicarbonato de sodio para crear una superficie calcárea que es un sustrato más apropiado. Al usar el cabo de polipropileno sin tratar, no se pudieron formar DFS en su superficie.

Las conclusiones de este análisis se contraponen a las de Arbaiza S. (2016), que después de 20 días evidenció la formación de DFS en todas las poblaciones de estudio y también la investigación de Sáez y Macchiavello (2018), quienes apreciaron que el periodo de cohesión de DFS fue de 25 días. 
Bulboa et al. (2005), a través de los experimentos de cultivos en mar, observaron, que esta especie tiene la cualidad de formar un DFS sobre sustrato artificial, sujetando los talos de C. chamissoi al sustrato y evitando el desprendimiento, como estrategia de sobrevivencia en el tiempo, por lo que este estudio realizó el primer intento de corroborar dicha afirmación, utilizando el cabo de polipropileno.

Según Ernesto Pariona y Patricia Gil-Kodaka (2011) quienes realizaron experimentos de colonización en la playa Mendieta, Reserva Nacional de Paracas, el establecimiento y predominio de C. chamissoi sobre los sustratos calcáreos se dio de manera preferente. Asimismo, esto explicaría el hecho de que en el Tratamiento $C$ el cabo de propileno no haya sido un buen sustrato para la propagación de C. chamissoi. Para el Tratamiento B se estimó el tiempo de cicatrización de tejido fraccionado de frondas de C. chamissoi, a 15 días del inicio del acondicionamiento en sustrato, produciendo brotes primarios. Al día 20 se incrementaron los brotes y aparecieron nuevas ramificaciones en las frondas. En este lapso de tiempo la formación de DFS fue mínima, formándose nuevamente frondas iniciales que permitieron continuar con la siguiente etapa del estudio, cuando se inspeccionó que cada 10 días había un incremento de longitud.

Los resultados ratifican el estudio realizado por Saez, F., D. Yañez y J. Macchiavello (2015), indicándose que el periodo de sellado del tejido dañado fue posterior a 15 días. Con el estudio realizado cicatrización en frondas tetrasporofíticas y gametofíticas femeninas de C. chamissoi (Gigartinales, Rhodophyta).

\subsection{Crecimiento de C. chamissoi "Yuyo"}

La evaluación comparativa de crecimiento vegetativo en el presente estudio mostró un promedio entre los tratamientos con diferencias estadísticamente significativas $(p<0.05)$, para los tratamientos (A), (B) y $(P)$.

Previamente se realizó la prueba de normalidad para los tratamientos, obteniendo como resultado que los datos se encuentran dentro de los parámetros normales. En este caso se aplicó la prueba de normalidad de Shapiro - Wilk,

Tabla 1. Crecimiento promedio en longitud de fronda ( $\mathrm{mm}$.)

\begin{tabular}{|c|c|c|c|c|}
\hline TRATAMIENTO & A & B & C & P \\
\hline DÍA 10 & 1.4 & 2.3 & - & 1.3 \\
\hline DÍA 20 & 2.3 & 3.2 & - & 2.2 \\
\hline DÍA 30 & 3.0 & 4.1 & - & 2.9 \\
\hline DÍA 40 & 3.9 & 4.8 & - & 3.5 \\
\hline
\end{tabular}

Fuente: Elaboración propia.

En el experimento observamos diferencias de crecimiento, donde el tratamiento (B) sobresalió con relación a los tratamientos(A) y del Grupo Control (P). La tasa de crecimiento en general, para los 40 días de cultivo, es mayor ( $4.8 \mathrm{~mm}$ ), en comparación a los demás tratamientos, que mostraron crecimientos de $3.9 \mathrm{~mm}(\mathrm{~A})$ y $3.5 \mathrm{~mm}(\mathrm{P})$.

En consecuencia, se llevó a cabo un análisis comparativo en tiempo y crecimiento. A continuación se presenta una tabla con los resultados del análisis de varianza (tabla 2). 
Tabla 2. Análisis de varianza a 10, 20, 30 y 40 días

\begin{tabular}{|c|c|c|c|c|c|c|c|}
\hline \multicolumn{8}{|c|}{ ANÁLISIS DE VARIANZA A 10 DÍAS } \\
\hline \multirow{2}{*}{$\begin{array}{l}\text { Fuente de } \\
\text { Variación }\end{array}$} & \multirow{2}{*}{$\begin{array}{c}\text { Grados de } \\
\text { Libertad }\end{array}$} & \multirow{2}{*}{$\begin{array}{c}\text { Suma de } \\
\text { cuadrados }\end{array}$} & \multirow{2}{*}{$\begin{array}{l}\text { Cuadrados } \\
\text { Medios }\end{array}$} & \multirow{2}{*}{$F_{\text {calc }}$} & \multicolumn{2}{|c|}{$F_{\text {tablas }}$} & \multirow{2}{*}{$p$} \\
\hline & & & & & 0.05 & 0.01 & \\
\hline Tratamiento & 2 & 1.4 & 0.7 & 3.58 & 3.06 & 4.26 & 0.0013 \\
\hline Error & 6 & 1.2 & 0.2 & & & & \\
\hline Total & 8 & 2.7 & & & & & \\
\hline \multicolumn{2}{|c|}{ Coeficiente de Variabilidad } & \multicolumn{2}{|c|}{26.2} & \multicolumn{2}{|c|}{ Promedio } & \multicolumn{2}{|c|}{1.7} \\
\hline \multicolumn{8}{|c|}{ ANÁLISIS DE VARIANZA A 20 DÍAS } \\
\hline \multirow{2}{*}{$\begin{array}{l}\text { Fuente de } \\
\text { Variación }\end{array}$} & \multirow{2}{*}{$\begin{array}{l}\text { Grados de } \\
\text { Libertad }\end{array}$} & \multirow{2}{*}{$\begin{array}{c}\text { Suma de } \\
\text { cuadrados }\end{array}$} & \multirow{2}{*}{$\begin{array}{l}\text { Cuadrados } \\
\text { Medios }\end{array}$} & \multirow{2}{*}{$F_{\text {calc }}$} & \multicolumn{2}{|c|}{$F_{\text {tablas }}$} & \multirow{2}{*}{$\mathrm{p}$} \\
\hline & & & & & 0.05 & 0.01 & \\
\hline Tratamiento & 2 & 1.6 & 0.8 & 24.65 & 3.06 & 4.26 & 0.0000 \\
\hline Error & 6 & 0.2 & 0.0 & & & & \\
\hline Total & 8 & 1.7 & & & & & \\
\hline \multicolumn{2}{|c|}{ Coeficiente de Variabilidad } & \multicolumn{2}{|c|}{6.9} & \multicolumn{2}{|c|}{ Promedio } & \multicolumn{2}{|c|}{2.6} \\
\hline \multicolumn{8}{|c|}{ ANALISIS DE VARIANZA A 30 DÍAS } \\
\hline \multirow{2}{*}{$\begin{array}{l}\text { Fuente de } \\
\text { Variación }\end{array}$} & \multirow{2}{*}{$\begin{array}{l}\text { Grados de } \\
\text { Libertad }\end{array}$} & \multirow{2}{*}{$\begin{array}{l}\text { Suma de } \\
\text { cuadrados }\end{array}$} & \multirow{2}{*}{$\begin{array}{l}\text { Cuadrados } \\
\text { Medios }\end{array}$} & \multirow[t]{2}{*}{$F_{\text {calc }}$} & \multicolumn{2}{|c|}{$F_{\text {tablas }}$} & $p$ \\
\hline & & & & & 0.05 & 0.01 & \\
\hline Tratamiento & 2 & 2.4 & 1.2 & 16.58 & 3.06 & 4.26 & 0.0000 \\
\hline Error & 6 & 0.4 & 0.1 & & & & \\
\hline Total & 8 & 2.8 & & & & & \\
\hline Coeficient & Variabilidad & & & Pro & edio & & 4 \\
\hline & & NALISIS DE & RIANZA A 40 & AS & & & \\
\hline Fuente de & Grados de & Suma de & Cuadrados & $F_{\text {calc }}$ & & & $p$ \\
\hline variacion & ertad & cuadrados & $\mathrm{Meo}$ & & 0.05 & 0.01 & \\
\hline Tratamiento & 2 & 1.7 & 0.8 & 8.76 & 3.06 & 4.26 & 0.0000 \\
\hline Error & 6 & 0.6 & 0.1 & & & & \\
\hline Total & 8 & 2.2 & & & & & \\
\hline Coeficient & Variabilidad & & & Pro & edio & & 2 \\
\hline
\end{tabular}

Fuente: Elaboración propia, basada en el registro de datos de crecimiento en longitud.

Dado que en todos los análisis de varianza se encontraron diferencias altamente significativas ( $p<0.01)$, y coeficientes de variabilidad altos (> 5\%), se aplicó la prueba de Tukey al $1 \%$, para establecer las diferencias estadísticas significativas.

A continuación mostramos los resultados de la prueba Tukey, para cada análisis de varianza, ya antes detallado. 
Tabla 3. Prueba de Tukey para varianza a 10, 20,30 y 40 días

\begin{tabular}{|c|c|c|c|}
\hline \multicolumn{4}{|c|}{ PRUEBA TUKEY EN 10 DÍAS } \\
\hline Nro. De Orden & Tratamiento & Rendimiento Promedio & Sig. \\
\hline 1 & A & 1.44 & A \\
\hline 2 & $\mathrm{~B}$ & 2.28 & b>ap. \\
\hline 3 & $P$ & 1.43 & $P$ \\
\hline \multicolumn{4}{|c|}{ PRUEBA TUKEY EN 20 DÍAS } \\
\hline Nro. De Orden & Tratamiento & Rendimiento Promedio & Sig. \\
\hline 1 & A & 2.28 & a \\
\hline 2 & $\mathrm{~B}$ & 3.15 & $\mathrm{~b}$ \\
\hline 3 & $P$ & 2.26 & a \\
\hline \multicolumn{4}{|c|}{ PRUEBA TUKEY EN 30 DÍAS } \\
\hline Nro. De Orden & Tratamiento & Rendimiento Promedio & Sig. \\
\hline 1 & A & 3.05 & a \\
\hline 2 & B & 4.12 & $\mathrm{~b}$ \\
\hline 3 & $P$ & 3.02 & a \\
\hline \multicolumn{4}{|c|}{ PRUEBA TUKEY EN 40 DÍAS } \\
\hline Nro. De Orden & Tratamiento & Rendimiento Promedio & Sig. \\
\hline 1 & A & 3.94 & a \\
\hline 2 & B & 4.78 & $b$ \\
\hline 3 & $P$ & 3.81 & a \\
\hline
\end{tabular}

Fuente: Elaboración propia, basada en el registro de datos de crecimiento en longitud.

El mejor crecimiento obtenido en los 40 días de cultivo en laboratorio, fue en el tratamiento (B), con una tasa de crecimiento de $4.78 \mathrm{~mm}$. $\pm 0.12 \mathrm{~mm}$./día y cultivada en sustrato artificial (malla de fruta), en comparación al tratamiento (A), en el cual se observó una tasa de crecimiento de $3.94 \mathrm{~mm}$. $\pm 0.10 \mathrm{~mm}$. /día, cultivada en valva de almeja; y en el grupo control (P), se observó una tasa de crecimiento diario de $3.70 \mathrm{~mm}$. \pm 0,09 mm. /día (Arbaiza et al. 2015; Bulboa et al. 2013).

Sin embargo, Arbaiza et al. (2015) obtuvieron un valor promedio de crecimiento y desarrollo muy heterogéneo entre las 4 iteraciones experimentales. La talla final fue T1: 2.15 mm, T2: 1.01 mm, T3: $1.13 \mathrm{~mm}$ y T4: $0.7 \mathrm{~mm}$., indicando que es muy voluble el crecimiento. Mientras que los resultados obtenidos por Bulboa et al. (2013) registraron variaciones entre 0,2 $\pm 0,03$ y 3,5 $\pm 1,2 \mathrm{~mm}$, en cultivos sobre sustrato natural, y en el sustrato artificial varió entre 0,8 $\pm 0,2$ y 5,4 $\pm 0,1 \mathrm{~mm}$.

\subsection{Supervivencia de C. chamissoi}

El análisis comparativo de supervivencia en las unidades de estudio entre los tratamientos, señala estimaciones promedio por periodo de control. 
A los datos de supervivencia obtenidos, se le aplicó un análisis de varianza en el periodo de estudio por tratamiento, que se presenta en la tabla que se muestra a continuación.

Tabla 4. Análisis de varianza en el periodo de estudio por tratamiento

\begin{tabular}{|c|c|c|c|c|c|c|c|}
\hline \multicolumn{8}{|c|}{ ANÁLISIS DE VARIANZA A 10 DÍAS } \\
\hline \multirow{2}{*}{$\begin{array}{l}\text { Fuente de } \\
\text { Variación }\end{array}$} & \multirow{2}{*}{$\begin{array}{c}\text { Grados de } \\
\text { Libertad }\end{array}$} & \multirow{2}{*}{$\begin{array}{c}\text { Suma de } \\
\text { cuadrados }\end{array}$} & \multirow{2}{*}{$\begin{array}{l}\text { Cuadrados } \\
\text { Medios }\end{array}$} & \multirow{2}{*}{$F_{\text {calc }}$} & \multicolumn{2}{|c|}{$F_{\text {tablas }}$} & \multirow{2}{*}{$p$} \\
\hline & & & & & 0.05 & 0.01 & \\
\hline Tratamiento & 2 & 1468.7 & 734.3 & 4.49 & 2.43 & 3.5 & 0.0000 \\
\hline Error & 6 & 981.3 & 163.6 & & & & \\
\hline Total & 8 & 2450.0 & & & & & \\
\hline \multicolumn{2}{|c|}{ Coeficiente de Variabilidad } & 40.4 & \multicolumn{3}{|c|}{ Promedio } & \multicolumn{2}{|c|}{31.7} \\
\hline \multicolumn{8}{|c|}{ ANÁLISIS DE VARIANZA A 20 DÍAS } \\
\hline \multirow{2}{*}{$\begin{array}{l}\text { Fuente de } \\
\text { Variación }\end{array}$} & \multirow{2}{*}{$\begin{array}{c}\text { Grados de } \\
\text { Libertad }\end{array}$} & \multirow{2}{*}{ S.C } & \multirow{2}{*}{$\begin{array}{l}\text { Cuadrados } \\
\text { Medios }\end{array}$} & \multirow[t]{2}{*}{$F_{\text {calc }}$} & \multicolumn{2}{|c|}{$F_{\text {tablas }}$} & \multirow{2}{*}{$\mathrm{p}$} \\
\hline & & & & & 0.05 & 0.01 & \\
\hline Tratamiento & 2 & 3066.9 & 1533.4 & 16.24 & 2.43 & 3.5 & 0.0000 \\
\hline Error & 6 & 566.7 & 94.4 & & & & \\
\hline Total & 8 & 3633.6 & & & & & \\
\hline \multicolumn{2}{|c|}{ Coeficiente de Variabilidad } & 38.7 & \multicolumn{3}{|c|}{ Promedio } & \multicolumn{2}{|c|}{25.1} \\
\hline \multicolumn{8}{|c|}{ ANÁLISIS DE VARIANZA A 30 DÍAS } \\
\hline \multirow{2}{*}{$\begin{array}{l}\text { Fuente de } \\
\text { Variación }\end{array}$} & \multirow{2}{*}{$\begin{array}{c}\text { Grados de } \\
\text { Libertad }\end{array}$} & \multirow{2}{*}{$\begin{array}{c}\text { Suma de } \\
\text { cuadrados }\end{array}$} & \multirow{2}{*}{$\begin{array}{l}\text { Cuadrados } \\
\text { Medios }\end{array}$} & \multirow{2}{*}{$F_{\text {calc }}$} & \multicolumn{2}{|c|}{$F_{\text {tablas }}$} & \multirow{2}{*}{$p$} \\
\hline & & & & & 0.05 & 0.01 & \\
\hline Tratamiento & 2 & 2038.6 & 1019.3 & 16.15 & 2.43 & 3.5 & 0.0156 \\
\hline Error & 6 & 378.7 & 63.1 & & & & \\
\hline Total & 8 & 2417.2 & & & & & \\
\hline \multicolumn{2}{|c|}{ Coeficiente de Variabilidad } & 38.2 & & omedio & & & \\
\hline & & ANÁLISIS DE & ARIANZA A $4 C$ & DÍAS & & & \\
\hline Fuente de & Grados de & Suma de & Cuadrados & $\boldsymbol{F}_{\text {calc }}$ & & & $p$ \\
\hline Iacion & er & cuadrados & M & & 0.05 & 0.01 & \\
\hline Tratamiento & 2 & 1851.9 & 925.9 & 14.20 & 2.43 & 3.5 & 0.0066 \\
\hline Error & 6 & 391.3 & 65.2 & & & & \\
\hline Total & 8 & 2243.2 & & & & & \\
\hline Coeficiente & Variabilidad & 41.1 & & omedio & & & \\
\hline
\end{tabular}

Fuente: Elaboración propia, basada en el registro de supervivencia.

A los resultados del análisis de varianzas, con respecto al tratamiento y tiempo, se le aplicó respectivamente la prueba Tukey. 
Tabla 5. Prueba de Tukey para el anterior análisis de varianza (tabla 4)

\begin{tabular}{|c|c|c|c|}
\hline \multicolumn{4}{|c|}{ PRUEBA TUKEY EN 10 DÍAS } \\
\hline Nro. De Orden & Tratamiento & Rendimiento Promedio & Sig. \\
\hline 1 & A & 49.667 & a \\
\hline 2 & $\mathrm{~B}$ & 21.333 & $\mathrm{~b}$ \\
\hline 3 & $P$ & 24.00 & $b$ \\
\hline \multicolumn{4}{|c|}{ PRUEBA TUKEY EN 20 DÍAS } \\
\hline Nro. De Orden & Tratamiento & Rendimiento Promedio & Sig. \\
\hline 1 & $\mathrm{~A}$ & 32.33 & a \\
\hline 2 & $\mathrm{~B}$ & 21.00 & $b$ \\
\hline 3 & $\mathrm{P}$ & 22.00 & b \\
\hline \multicolumn{4}{|c|}{ PRUEBA TUKEY EN 30 DÍAS } \\
\hline Nro. De Orden & Tratamiento & Rendimiento Promedio & Sig. \\
\hline 1 & A & 23.33 & a \\
\hline 2 & $\mathrm{~B}$ & 20.00 & $\mathrm{~b}$ \\
\hline 3 & $P$ & 19.00 & $b$ \\
\hline \multicolumn{4}{|c|}{ PRUEBA TUKEY EN 40 DÍAS } \\
\hline Nro. De Orden & Tratamiento & Rendimiento Promedio & Sig. \\
\hline 1 & A & 22.00 & a \\
\hline 2 & $B$ & 18.66 & $b$ \\
\hline 3 & $P$ & 18.33 & $b$ \\
\hline
\end{tabular}

Fuente: Elaboración propia, basada en el registro de supervivencia.

Y se obtuvieron los siguientes resultados: Para el tratamiento A, se calculó en los primeros 10 días un promedio de 49 ejemplares, El día 20 el promedio rodeó un valor aproximado de 32 ejemplares. El día 30 se promedió un valor de 23 ejemplares. Y el día 40 se obtuvo un valor promedio de 22 ejemplares.

En cuanto al tratamiento $B$, precisamos que pasados los 10 días se obtuvo un valor promedio de 21 ejemplares, El día 20 el resultado promedio fue de 21 ejemplares; el día 30 su valor promedio resultante fue 20. Y el día 40 se anotó un resultado promedio de 19 ejemplares.

Para el tratamiento $\mathrm{P}$, a los 10 días se obtuvo un valor aproximado de 21 ejemplares, El día 20 registró 21 ejemplares; el día 30 se indicó un valor promedio de 20 ejemplares. Y el día 40 se mostró un valor promedio de 18 ejemplares, observando una tendencia descendente, lo cual se especifica a continuación.

En este estudio se reconocieron, en los tratamientos $(B)$ y $(P)$, elevadas tasas de supervivencia, comparadas con el tratamiento (A), observándose en los primeros 20 días de cultivo una notable diferencia en relación a los tratamientos B y P, lo que reflejaría una alta densidad de discos de fijación secundaria en el tratamiento (A). Estos resultados evidencian que la supervivencia promedio para el tratamiento (A) es de $74.39 \%$ y para los tratamientos (B) y (P) es de $85.75 \%$. Con relación a lo anterior, Macchiavello et al. (2013) también reportaron que las altas tasas de supervivencia de C. chamissoi, tanto en sustratos naturales como artificiales, se debe a que los DFS se van formando continuamente, lo que promueve la incorporación de juveniles y aumenta el anclaje de las algas en crecimiento sobre los sustratos. 
Por otro lado, Bulboa et al. (2013) describieron que las tasas de supervivencia de DFS, después de 40 días, variaron entre $60 \pm 12$ y $100 \%$. En tallos unidos en sustrato natural, los valores oscilaron entre $89 \pm 3$ y $98 \pm 3 \%$ para sustrato artificial; sin embargo, no se puede atribuir que la supervivencia dependió directamente del tipo de sustrato utilizado, considerando que en cada tratamiento se utilizó sustratos distintos, además se trabajó en condiciones controladas, lo que es muy distinto de las condiciones naturales. Por lo que el mayor densidad de DFS en el tratamiento A puede estar promovido, no solo por el propio sustrato utilizado (valvas de almejas), sino por la posible presencia en el mismo de micronutrientes trazas (Bishop, 2011). Asimismo, Pariona y Gil-Kodaka (2011) observaron que la C. chamissoi crece de mejor manera en sustratos calcáreos, como en este caso las valvas de almejas.

\section{Conclusiones y recomendaciones}

Se determinó que la mejor tasa de crecimiento obtenido en los 40 días de cultivo, fue en el tratamiento (B), con un valor de $4,78 \mathrm{~mm} . \pm 0.12 \mathrm{~mm}$. /día, el mismo que fue cultivado en sustrato artificial (malla de fruta), a partir de algas fragmentadas.

La supervivencia más alta fue en el tratamiento (B) y (P) de $85.75 \%$ ambos y de $74.39 \%$ en el tratamiento $(A)$.

También se determinó que el uso de cabo de propileno no permite el desarrollo de discos de fijación secundaria, por lo que no se recomienda para su uso en cultivo de $C$. chamissoi.

En relación a lo concluido, se recomienda realizar un estudio sobre el cultivo de la C. chamissoi, mediante la macro fragmentación de frondas in vitro. Asimismo, profundizar trabajos de investigación relacionados a los inductores en sustratos artificiales y su aplicación en el cultivo de C. chamissoi.

También se propone promover el escalamiento de experiencias similares a este estudio, con el objetivo de dar valor agregado a las algas rojas, considerando su potencial reproductivo y factibilidad de cultivo. Realizar trabajos de investigación donde se incluyan variables económicas, que permitan determinar los índices de rentabilidad de los cultivos marinos.

\section{Referencias}

Alveal, K. R. I. S. L. E. R. (2001). Estrategias reproductivas de Rhodophyta y sus nexos con la biodiversidad. Sustentabilidad de la biodiversidad. Un problema actual: bases científico-técnicas. Teorizaciones y proyecciones, 367-388.

Arbaiza, S., (2016). Viabilidad reproductiva para el cultivo de Chondracanthus chamissoi proveniente de tres poblaciones del litoral Peruano, p. 1-114. Lima, Perú.

Arbaiza, S., Gil, P. y Arakaki, N. (2015). Fijación de Carposporas y formación de discos de fijación secundaria de Chondracanthus chamissoi "yuyo" (Paiján, Perú) en condiciones Semi Controladas de Laboratorio 1(1), 1.

Arbaiza, S., Gil-Kodaka, P., Arakaki, N. y Alveal, K. (2019). Primeros estadios de cultivo a partir de carpósporas de Chondracanthus chamissoi de tres localidades de la costa peruana. Revista de Biología Marina y Oceanografía 54(2), 204-213.

Bishop, K. (2011). The Effect of Substrate Properties on the Attachment and Reproduction of Diatoms. [Seniors dissertation, William \& Mary University]. https://bit.ly/3ITZ4fF

Bulboa, C., \& Macchiavello, J. (2006). Cultivation of Cystocarpic, Tetrasporic and Vegetative Fronds of Chondracanthus chamissoi (Rhodophyta, Gigartinales) on Ropes at two Localities in Northern Chile. Investigaciones Marinas 34, 151-154. 
Bulboa, C., Macchiavello, J., Oliveira, E. y Fonck, E. (2005). First Attempt to Cultivate the Carrageenan Producing Seaweed Chondracanthus chamissoi (Rhodophyta; Gigartinales) in Northern Chile. Aquaculture Research 36, 1069-1074.

Bulboa, C., Veliz, K., Saez, F. et al. (2013). A New Method for Cultivation of the Carragenophyte and Adible Red Seaweed Chondracanthus chamissoi Based on Secondary Attachment Disc: Development Inoutdoor Tanks. Aquaculture Research 94, 410-411.

Macchiavello, J., Bulboa, C., \& Edding, M. (2003). Vegetative Propagation and Spore Recruitment in the Carrageenophyte Chondracanthus chamissoi (Rhodophyta, Gigartinales) in northern Chile. Phycol. Res., 51, 45-50.

Macchiavello, J., Bulboa, C., Sepúlveda, C. et al. (2013). Alternativas tecnológicas para el cultivo de Chondracanthus chamissoi en la zona norte de Chile a partir de discos de fijación secundaria. Manual de Cultivo de Chondracanthus chamissoi (Chicorea de mar). Fondef-HUAM AQ08I-1028.

Pariona, E. y Gil-Kodaka, P. (2011). Colonización de Chondracanthus chamissoi (Rhodophyta, Gigartinales) sobre sustratos calcáreos en Playa Mendieta, 59 Reserva Nacional de Paracas. [Revista científica] doi: 10.21704/ac.v76i2.854, lima.

Saez, F.; Macchiavello, J.; Fonck, E. y Bulboa, C. (2008). The Role of the Secondary Attachment Disc in the Vegetative Propagation of Chondracanthus chamissoi (Gigartinales, Rhodophyta). Aquatic Botany 89, 63-65.. 